\title{
Trigeminal neuralgia caused by fibrous bands: case illustration
}

\author{
Yoshifumi Mizobuchi, MD, PhD, Kenji Shouno, MD, Kohei Nakajima, MD, PhD, and \\ Shinji Nagahiro, MD, PhD
}

Department of Neurosurgery, Institute of Health Biosciences, The University of Tokushima Graduate School, Tokushima, Japan

http://thejns.org/doi/abs/10.3171/2014.12.JNS1463

KEY WORDS trigeminal neuralgia; fibrous band; epidermoid tumor; peripheral nerve

$\mathrm{W}$ E describe a rare case of trigeminal neuralgia caused by a fibrous band after epidermoid tumor resection. A 31-year-old woman presented with right-sided facial pain. MRI showed an abnormal enhancement indicating a neoplasm in the right cerebellopontine cistern. Resection was performed, and the tumor was completely removed (Fig. 1 left). The final diagnosis was epidermoid tumor. Two years after surgery, the patient experienced a gradual return of pain that became refractory to medication. She was referred to our hospital, where MRI revealed notches in the proximal and distal sides of the trigeminal nerve not seen on the immediate postoperative MR image (Fig. 1 right). On images obtained intraoperatively, we observed no offending artery that compressed a nerve or distortion of the trigeminal nerve. Instead, we found fibrous bands constricting the trigeminal nerve at proximal and distal sites (Fig. 2 left). After the fibrous bands were cut, the patient's neuralgia was relieved, and no other sensory deficit occurred (Fig. 2 right).

In patients presenting with typical trigeminal neuralgia but with an MRI-documented notch seen in the trigeminal nerve, the condition may be caused by an unusual type of trigeminal nerve constriction. ${ }^{1,3,4}$ To the best of our knowledge, trigeminal neuralgia due to a fibrous band has been reported only once in the literature. ${ }^{2}$
In conclusion, a fibrous band thus seems to be an extremely rare cause of trigeminal neuralgia. Therefore, surgery to release the constricted nerve should be considered even if MRI shows no apparent tumor recurrence or offending artery around the trigeminal nerve.

\section{References}

1. Bathla G, Hegde AN: The trigeminal nerve: an illustrated review of its imaging anatomy and pathology. Clin Radiol 68:203-213, 2013

2. Kimura T, Sameshima T, Morita A: Trigeminal neuralgia caused by a fibrous ring around the nerve. J Neurosurg 116:741-742, 2012

3. Leclercq D, Thiebaut JB, Heran F: Trigeminal neuralgia. Diagn Interv Imaging 94:993-1001, 2013

4. Love S, Coakham HB: Trigeminal neuralgia: pathology and pathogenesis. Brain 124:2347-2360, 2001

\footnotetext{
Author Contributions

Conception and design: Mizobuchi. Acquisition of data: Mizobuchi. Analysis and interpretation of data: Mizobuchi. Drafting the article: Mizobuchi. Critically revising the article: all authors. Reviewed submitted version of manuscript: all authors. Approved the final version of the manuscript on behalf of all authors: Mizobuchi. Administrative/technical/material support: Nagahiro. Study supervision: Nagahiro.
} 

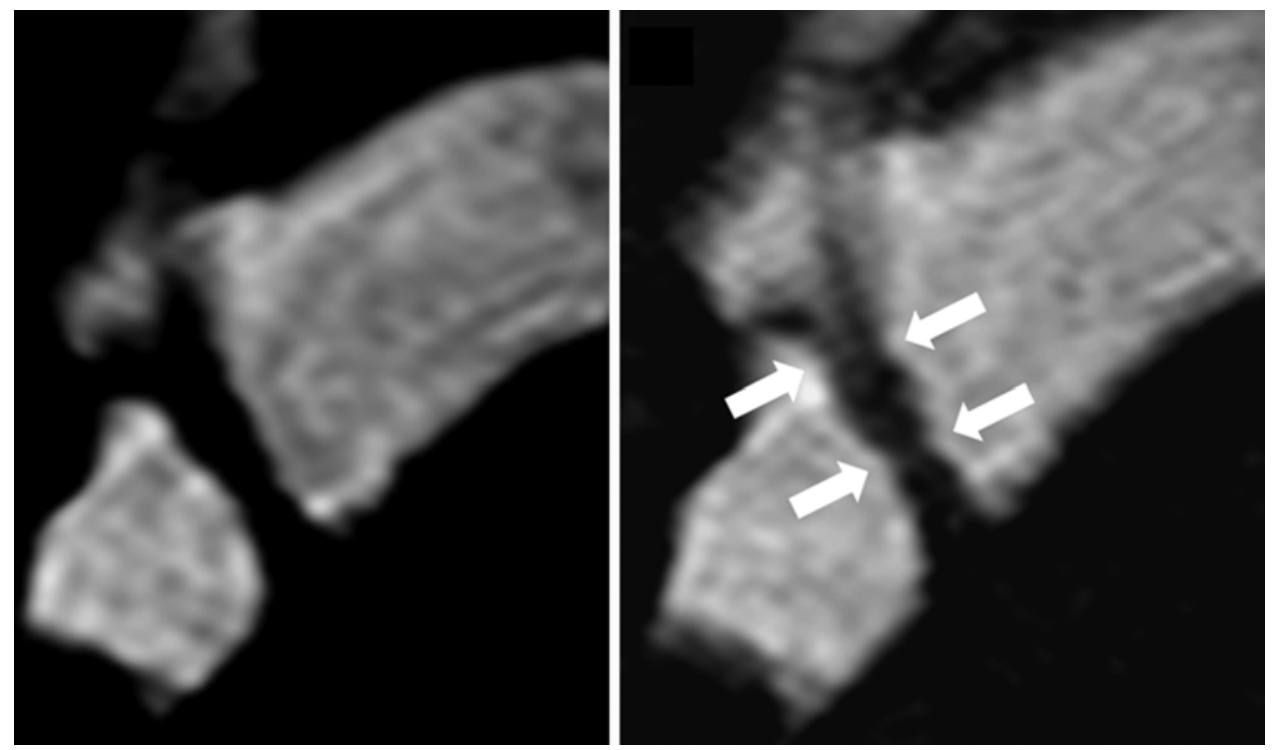

FIG. 1. Left: Postoperative 3D FIESTA image showing that the epidermoid tumor has been completely removed from the right cerebellopontine cistern. Right: 3D FIESTA image obtained 2 years after surgery demonstrating no apparent tumor recurrence. The arrows indicate notches in the right trigeminal nerve at the proximal and distal sites. The central part of the right trigeminal nerve has become barrel shaped.
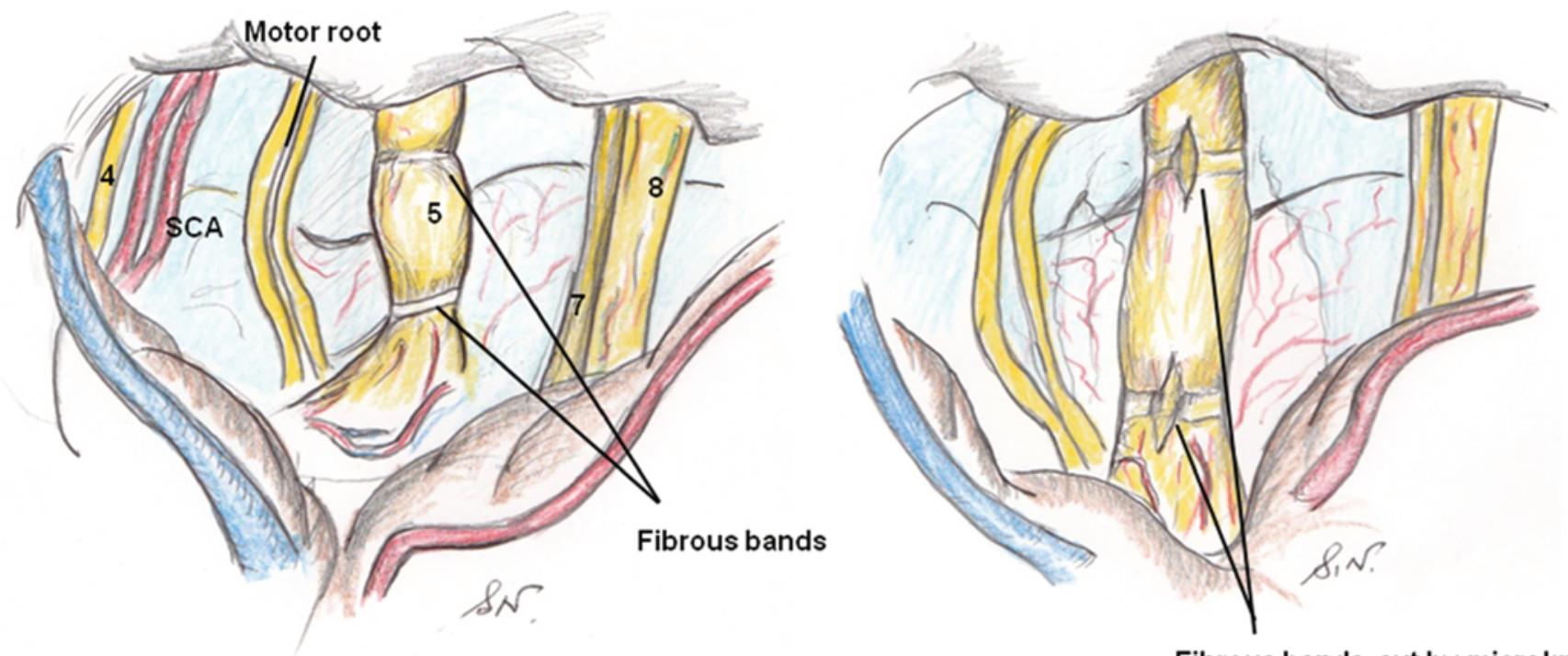

Fibrous bands, cut by microknife

FIG. 2. Left: Illustration of the diseased trigeminal nerve, showing an absence of both distortion and arterial loops capable of compressing the nerve. Close inspection revealed that the nerve was constricted by two fibrous circumferential bands. Right: IIlustration of the fibrous bands after they were cut using a microknife. Immediately after cutting, the constricted parts expanded slightly. SCA = superior cerebellar artery. Copyright Yoshifumi Mizobuchi. Published with permission. 